\title{
Quantifying Heterogeneity in the Pu-Ga System
}

\author{
Charles C. Davis and Rollin E. Lakis
}

Los Alamos National Laboratory, Mailstop E-574, P.O. Box 1663, Los Alamos NM 87545.

Plutonium $(\mathrm{Pu})$, atomic number of 94, is known to have 6 allotropes (crystalline forms) at atmospheric pressure between room temperature and $640^{\circ} \mathrm{C}$, the melting point of $\mathrm{Pu}$. The alphaphase (monoclinic), thermodynamically stable at room temperature, is hard and brittle making it a less than ideal metal for various metallurgical processes. Elements such as aluminum (Al) or gallium (Ga) may be added to Pu to stabilize the FCC delta-phase at room temperature. This phase is ductile, and a much more suitable material for the manufacture of complex parts. During the solidification of Pu-Ga alloys (nominal $1 \mathrm{wt} \% \mathrm{Ga}$ ) from the melt, the first delta-phase material is formed Ga-rich. This is due to the shape of the delta-epsilon phase field and rapid Ga diffusion at this temperature (Figure 1). The resulting microstructure is heavily cored with high $\mathrm{Ga}$ concentrations in the center of every grain and decreasing concentrations near grain boundaries as depicted in the optical micrograph in Figure 2. The extent of Ga segregation can be affected by controlling the cooling rate through the delta-epsilon phase field and by homogenizing the alloy in the high temperature range of the delta phase field. It is important that the Ga be relatively homogenous to ensure that during subsequent processing all the material remains stabilized in the delta phase, and does not transform to alpha-phase under the influence of stress and temperature.

A common analytical approach would be to measure quantitative line profiles through a few grains. These line profiles would provide a quantitative measure of solute concentration through those grains, but it is difficult to make comparisons from one sample to another or even in the same sample following thermal processing. Also, these line scans have not proven to be a reproducible measure of delta-phase stability against thermal and mechanical treatments.

By applying an analysis of variance method, using a random effects model for a nested experimental design, a measure of Ga heterogeneity can be made by comparing measured solute compositional variances in the sample. The variances compare the solute measurements from point to point, area to area and replicas of each point. The variation between areas is an indication of solute distribution similarity within the sample. Variations of replica measurements are an indication of equipment reproducibility and variations in point to point measurements would indicate the amount or the presence of coring in the sample.

$\mathrm{Pu}-\mathrm{Ga}$ samples were heat treated at $450^{\circ} \mathrm{C}$ for $0.1,12$ and $200 \mathrm{hrs}$. The analysis consisted of 5 areas of interest on the sample, 2 areas near the surfaces of the sheet, and 1 area in the middle. Each area had a grid of 50 points and 3 replicate measurements per point (Figure 3 ). Figure 4 compares the standard deviations of the variances, noting that the standard deviations are relatively small in magnitude $10^{-8}$. The standard deviation of the replica measurements is an indication of the precision of the measurements. The standard deviations between the 5 areas indicate that the sample heterogeneity is similar in those areas, and is of the same order as the measurement precision. The differences in the standard deviations in the point-to-point measurements from the $0.1,12$ and $200 \mathrm{hr}$ heat-treat times indicates a definite coring of the microstructure and increased point-to-point compositional heterogeneity. Future experiments will be performed to optimize the use of instrument time to improve some aspects of the measurement statistics; less time measuring replica precision and more time measuring point-to-point heterogeneity. In addition, work to correlate heterogeneity measurements to a measure of phase stability, will be attempted. 
Figure 1. Pu-Ga Phase Diagram

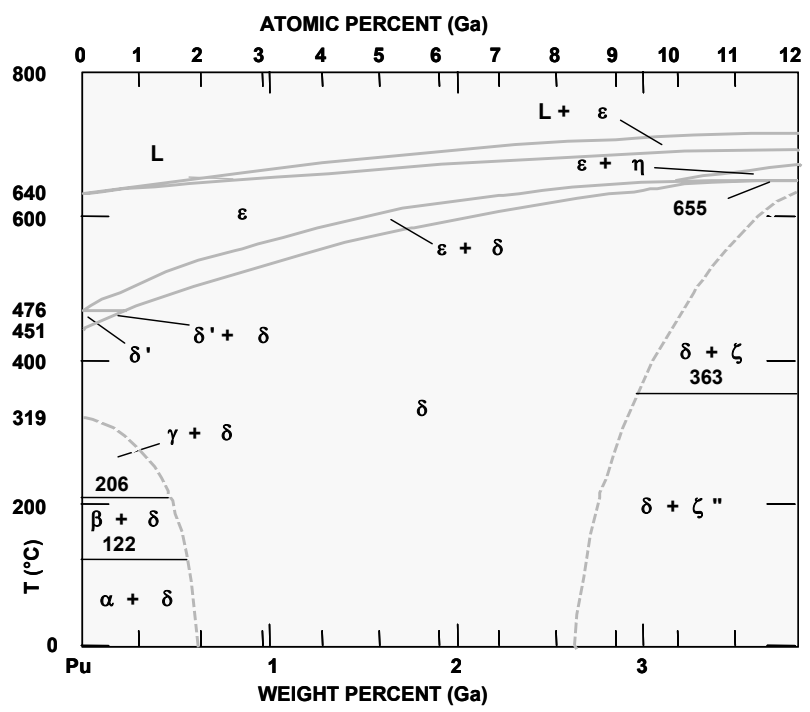

Figure 3 Variance Measurements

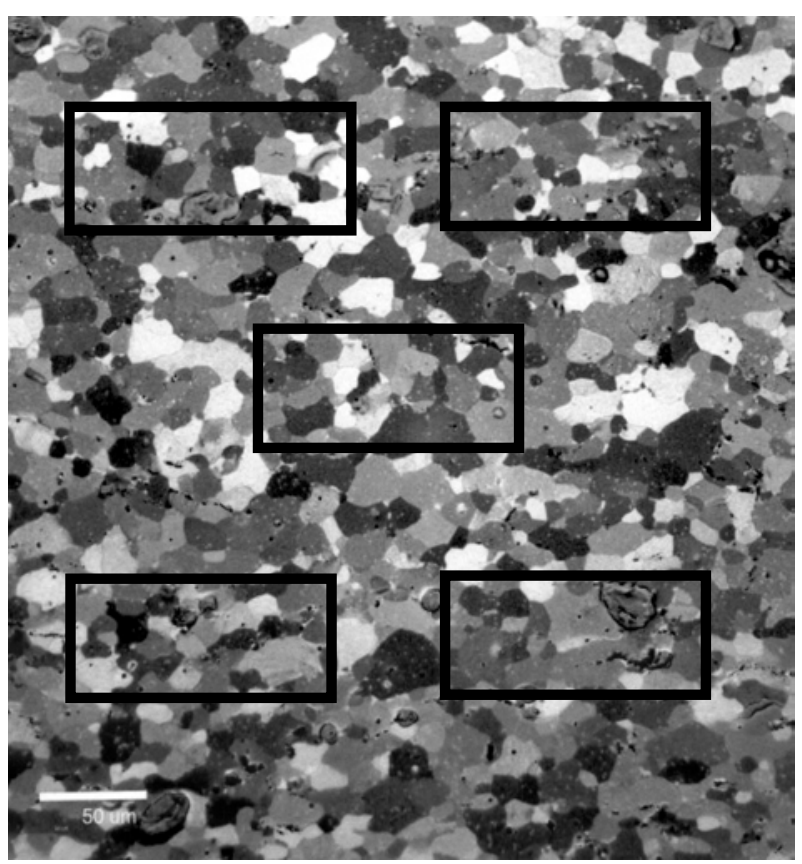

Figure 2. Cored Microstructure

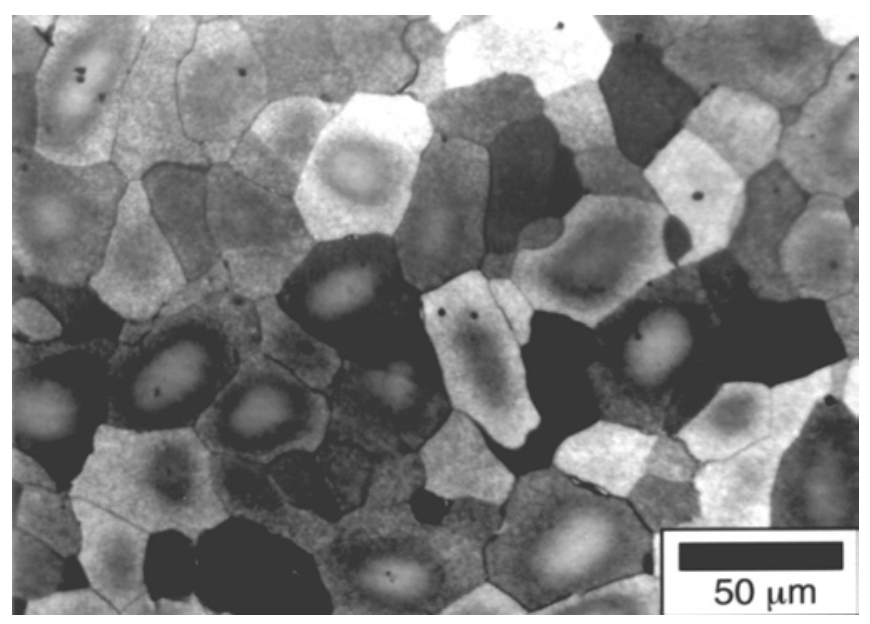

Figure 4

\section{Uncertainty Results}

\begin{tabular}{|c|c|c|c|c|}
\hline \multicolumn{5}{|c|}{ Standard Deviation $10^{-8}$} \\
\hline Sample & $\begin{array}{c}\text { Heat } \\
\text { Treatment } \\
\text { Hours }\end{array}$ & $\begin{array}{c}\text { Standard } \\
\text { Deviation } \\
\text { Area }\end{array}$ & $\begin{array}{c}\text { Standard } \\
\text { Deviation } \\
\text { Points }\end{array}$ & $\begin{array}{c}\text { Standard } \\
\text { Deviation } \\
\text { Replica }\end{array}$ \\
\hline Sample 1 & 0.1 & 1.6 & 240 & 3.0 \\
\hline Sample 2 & 12 & 2.3 & 130 & 2.8 \\
\hline Sample 3 & 200 & 1.2 & 79 & 3.1 \\
\hline
\end{tabular}

